\title{
Editorial
}

Oncology

\section{A Unique Role for Retrospective Studies in Clinical Oncology}

\author{
Maurie Markman \\ Cancer Treatment Centers of America and Drexel University College of Medicine, Philadelphia, Pa., USA
}

There is a long-established tradition of retrospective studies in clinical cancer investigation. Single institutional data or large multicenter efforts examining past experiences can serve both as hypothesis-generating elements for a future prospective clinical study, an idea to be explored in a translational laboratory research project, and even as confirmation of the results of a reported study in a more heterogeneous patient population.

Further, retrospective analyses can provide critically relevant data in populations known to be poorly represented in cancer clinical trials (e.g. the elderly and individuals with relevant comorbidities) and may identify adverse events potentially not recognized in the often highly homogenous groups of study participants. Finally, both the safety and the efficacy associated with longer observation periods and a more prolonged therapy than reported in many prospective clinical trials can be revealed through retrospective examinations of previously treated patients.

In the current issue of Oncology, Esposito et al. [1] present an interesting retrospective analysis of patients with recurrent clear-cell ovarian cancer, a most uncommon condition and one for which there are preciously limited data suggesting an effective therapeutic strategy. As a result, this paper represents a provocative example of yet another use of a retrospective study design.

In this retrospective analysis involving 72 patients who underwent second-line therapy at one of 20 Italian centers over a 16-year period, the investigators noted a small subgroup ( $\mathrm{n}=12)$ who received the antineoplastic agent gemcitabine and who appeared to have a higher rate of tumor response compared to individuals administered either topotecan or pegylated liposomal doxorubicin [1].

Of course, it must be emphasized that the very limited sample size does not permit any definitive conclusions regarding the relative utility of any individual strategy, including providing a truly meaningful 'objective response rate'. However, recognizing the rarity of this specific malignant condition (72 total patients identified in a period of 16 years at 20 centers), this retrospective experience will likely be of some value to individual oncologists needing to consider potential therapeutic options for a patient with recurrent clear-cell ovarian cancer. Further, in the event a multi-institutional prospective trial is ultimately undertaken in this most uncommon clinical setting, the results of this retrospective analysis should surely help to inform the planned study design.

Reference Esposito F, Cecere SC, Magazzino F, Katsaros
D, Ottaiano A, Gadducci A, Pisano C, Scalone
S, Rabaiotti E, Salutari V, Cormio G, Canuto
EM, Greggi S, Savarese A, Marinaccio M,
Scollo P, Santeufemia DA, Sacco C, Facchini
G, Pignata S: Second-line chemotherapy in
recurrent clear cell ovarian cancer: results
from the Multicenter Italian Trials in Ovarian
Cancer (MITO-9). Oncology 2014;86:351-
358. 